\title{
Massless fields in plane wave geometry
}

\author{
R.R. Metsaev \\ Department of Theoretical Physics, P.N. Lebedev Physical Institute, 117924, Leninsky \\ prospect 53, Moscow, Russia.
}

\begin{abstract}
Conformal isometry algebras of plane wave geometry are studied. Then, based on the requirement of conformal invariance, a definition of masslessness is introduced and gauge invariant equations of motion, subsidiary conditions, and corresponding gauge transformations for all plane wave geometry massless spin fields are constructed. Light cone representation for elements of conformal algebra acting as differential operators on wavefunctions of massless higher spin fields is also evaluated. Interrelation of plane wave geometry massless higher spin fields with ladder representation of $u(2,2)$ algebra is investigated.
\end{abstract}




\section{Introduction}

The plane wave [1] has been actively studied from various point of view in the context of both field theory (see [2] [3] [4] [5]) and string theory (see [6] [7] [8] [9] [10] [11] [12]). One of the remarkable properties of the plane wave is that it provides a non-perturbative solution to the classical equations of motion of superstring effective field theory. It is this property that has inspired a renewed interest in the plane wave in recent years. At present time a significant role of the plane wave is beyond doubt. Due to that, it is highly desirable to study the questions related to fields propagating in plane wave geometry (see [5]).

In this paper we study the following questions related to massless fields: (1) definition of masslessness in four-dimensional plane wave geometry; (2) gauge invariant equations of motion and corresponding gauge transformations as well as subsidiary conditions; (3) relation of plane wave geometry massless fields to ladder representations.

The paper is organized as follows. In Sec. II we shall review isometry algebras of various plane wave backgrounds and construct complete expressions for generators, i.e., corresponding orbital and spin parts. In Sec. III we shall consider conformal isometry algebras. We present complete manifest expressions for elements of conformal algebras, i.e., corresponding orbital, spin, and conformal boost parts. In Sec. IV, based on conformal invariance, we shall construct free equations of motion for all, lower as well as higher, massless spin fields. These equations we supplement by corresponding gauge transformations and subsidiary conditions. We study the wave equation of motion for massless field and demonstrate an existence a nonzero energy lowest value for a massless field. We construct also a light cone representation for elements of a conformal algebra acting as differential operators on a wavefunction of massless field. In Sec. V we shall demonstrate explicitly the manner in which the infinity chain of plane wave geometry massless spin fields $\lambda=0, \pm 1 / 2, \pm 1, \pm 3 / 2, \pm 2, \ldots$ (every state appears once) can be embedded into ladder representation of the $u(2,2)$ algebra. By using ladder representations we demonstrate also how Minkowski and plane wave geometry massless fields are related to each other. In Sec. VI we shall describe possible applications of our results. Appendices A and B detail certain mathematical manipulations.

\section{Isometry Algebras}

\section{A. General case}

The metric for a general gravitational-electromagnetic plane wave can be read as follows:

$$
d s^{2}=2 d u d v+2\left(f(u) \zeta^{2}+\bar{f}(u) \bar{\zeta}^{2}+F(u) \zeta \bar{\zeta}\right) d u^{2}-2 d \zeta d \bar{\zeta} .
$$

Note that (2.1) is sometimes called an exact plane wave. The metric (2.1) is written in coordinates $(u, v, \zeta, \bar{\zeta})$. In what follows it will be convenient to use the coordinates $x^{\mu}=\left(u, v, \zeta_{i}\right)$, where $\zeta_{i}$ are given by

$$
\zeta=\left(\zeta_{1}+\mathrm{i} \zeta_{2}\right) / \sqrt{2}, \quad \bar{\zeta}=\left(\zeta_{1}-\mathrm{i} \zeta_{2}\right) / \sqrt{2}
$$


All the variables $u, v$, and $\zeta_{i}$ range from $-\infty$ to $+\infty$. Throughout this paper, unless otherwise specified, we use the following notation and conventions: (1) the indices $i, j$, and $k$ run over 1,$2 ;(2) \zeta^{2}, \partial^{2}$, and $(\zeta \partial)$ stand for $\zeta_{i} \zeta_{i}, \partial_{i} \partial_{i}$, and $\zeta_{i} \partial_{i}$ respectively, where $\partial_{i}=\partial / \partial \zeta^{i}, \zeta_{i}=\zeta^{i}$.

In Ref. [11] (see also Ref.[2]) the various plane waves have been classified according to the number of Killing symmetries they possess:

$\begin{array}{cccc} & f(u) & F(u) & \operatorname{dim}(\text { Killing sym) } \\ I & 0 & F \neq 0 & 7 \\ I I & 0 & F(u) & 6 \\ I I I & f \neq 0 & F & 6 \\ I V & f(u) & F(u) & 5\end{array}$

where $f$ and $F$ without argument stand for $u$-independent constants. Note that the I and II cases (vanishing Weyl tensor) are usually called purely electromagnetic plane wave, while one of the particulars of III and IV when $F(u)=0$ (vanishing Ricci tensor) is called purely gravitational plane wave. The Killing vectors for $I-I V$ can be read as (see also Refs. [1] and [12])

$\begin{array}{ccccc}I & P_{v}, & P_{C}, & P_{u}, & J_{i j}, \\ I I & P_{v}, & P_{C}, & & J_{i j}, \\ I I I & P_{v}, & P_{C}, & P_{u}, & \\ I V & P_{v}, & P_{C}, & & \end{array}$

where

$$
\begin{gathered}
P_{v}=\partial_{v}, \quad P_{u}=\partial_{u}, \\
l\left(P_{C}\right)=C_{i}^{\prime} \zeta_{i} \partial_{v}+C_{i} \partial_{i}, \\
l\left(J_{i j}\right)=-\zeta_{i} \partial_{j}+\zeta_{j} \partial_{i},
\end{gathered}
$$

and prime indicates partial derivative with respect to $u$. Here $C_{i}$ satisfy the following second-order differential equation:

$$
C^{\prime \prime}+2 \bar{f}(u) \bar{C}+F(u) C=0,
$$

where $C=\left(C_{1}+\mathrm{i} C_{2}\right) / \sqrt{2}, \bar{C}=\left(C_{1}-\mathrm{i} C_{2}\right) / \sqrt{2}$. In (2.4), (2.5) and below a notation $l(G)$ is used to indicate the fact that corresponding expression on the rhs provides only the orbital part of algebra element $G$.

\section{B. I and II cases}

The I and II cases have a rotation Killing vector (2.5). Due to that we are able to introduce spin-s tensor fields $\Phi^{\mu_{1}, \ldots, \mu_{s}}$, where the spin $s$ can be associated with eigenvalue of $J_{i j}$. Since the expressions (2.3)-(2.5) provide only orbital parts they should be accompanied by corresponding spin parts. To derive spin parts we proceed as follows. First of 
all, we would like to pass to tangent space tensor fields. To do that we should introduce local a frame. One convenient choice is specified by the frame one-forms $\mathbf{e}^{A}=\mathbf{e}_{\mu}^{A} d x^{\mu}$ with

$$
\begin{aligned}
d s^{2} & =2 \mathbf{e}^{U} \mathbf{e}^{V}-\mathbf{e}^{i} \mathbf{e}^{i}, \\
\mathbf{e}^{U} & =d u \\
\mathbf{e}^{V} & =d v+\frac{1}{2} F(u) \zeta^{2} d u, \\
\mathbf{e}^{i} & =d \zeta^{i}
\end{aligned}
$$

The connection one-forms, defined by

$$
d \mathbf{e}^{A}+\omega_{B}^{A} \wedge \mathbf{e}^{B}=0, \quad \omega^{A B}=-\omega^{B A}
$$

are then given by

$$
\omega^{V i}=-F(u) \zeta^{i} e^{U} .
$$

Dual forms $e_{A}$ to (2.7), $e_{A}\left(\mathbf{e}^{B}\right)=\delta_{A}^{B}$, read as

$$
\begin{aligned}
& e_{U}=\partial_{u}-\frac{1}{2} F(u) \zeta^{2} \partial_{v}, \\
& e_{V}=\partial_{v}, \\
& e_{i}=\partial_{i} .
\end{aligned}
$$

Now we introduce the tangent space tensor fields in the usual manner:

$$
\Phi^{A_{1} \ldots A_{s}}=\mathbf{e}_{\mu_{1}}^{A_{1}} \ldots \mathbf{e}_{\mu_{s}}^{A_{s}} \Phi^{\mu_{1} \ldots \mu_{s}} .
$$

Then to avoid cumbersome tensor expressions we introduce creation and annihilation operators $a_{A}$ and $\bar{a}_{A}$ which satisfy

$$
\left[\bar{a}_{A}, a_{B}\right]=-\eta_{A B}, \quad \eta_{U V}=\eta_{V U}=1, \quad \eta_{i j}=-\delta_{i j},
$$

and construct a Fock space vector

$$
|\Phi\rangle=a_{A_{1}} \ldots a_{A_{s}} \Phi^{A_{1} \ldots A_{s}}|0\rangle, \quad \bar{a}_{A}|0\rangle=0 .
$$

Now the nonvanishing spin parts of isometry algebra under consideration are given by

$$
\begin{aligned}
& s\left(J_{i j}\right)=M_{i j}, \\
& s\left(P_{C}\right)=C_{i}^{\prime} M_{V i},
\end{aligned}
$$

where the elements $M_{A B}$ constitute $s o(3,1)$ algebra

$$
\left[M_{A B}, M_{C D}\right]=\eta_{A C} M_{D B}+\ldots
$$

(for details see Appendix A). 


\section{Conformal Isometry Algebras}

The purpose of this section is to get a manifest representation for elements of a conformal isometry algebra of plane wave geometry. This representation will be used throughout the paper. Before moving to the details, let us comment on the I and II cases. Since for these cases the Weyl tensor vanishes, the conformal algebra in question is isomorphic to the $s o(4,2)$ algebra. For these cases we also establish the isomorphism explicitly, i.e., we take each element of the conformal algebra calculated to an element of $s o(4,2)$ algebra taken in the Lorentz basis.

As usual we solve the equations for conformal Killing vectors

$$
\nabla_{\mu} \xi_{\nu}+\nabla_{\nu} \xi_{\mu}=\frac{1}{2} g_{\mu \nu} \nabla_{\rho} \xi^{\rho}
$$

The result of the solution can be summarized as follows:

$$
\begin{array}{ccccccc}
I & P_{v}, & P_{C}, & J_{i j}, & D, & T_{A}, K_{C}, K_{u}, \\
I I & P_{v}, & P_{C}, & J_{i j}, & D, & T_{A}, K_{C}, K_{u}, \\
I I I & P_{v}, & P_{C}, & & D, & P_{u}, & \\
I V & P_{v}, & P_{C}, & & D, &
\end{array}
$$

where

$$
\begin{aligned}
& l\left(T_{A}\right)=\frac{1}{4} \zeta^{2} A^{\prime \prime} \partial_{v}+A \partial_{u}+\frac{1}{2} A^{\prime}(\zeta \partial) \\
& l\left(K_{C}\right)=\zeta_{i}\left(v C_{i}^{\prime}-\frac{1}{2} \zeta^{2} F(u) C_{i}\right) \partial_{v}+C_{i} \zeta_{i} \partial_{u}+C_{i}^{\prime} \zeta_{i}(\zeta \partial)+\left(v C_{i}-\frac{1}{2} \zeta^{2} C_{i}^{\prime}\right) \partial_{i} \\
& l\left(K_{u}\right)=\left(v^{2}-\frac{1}{4} F(u)\left(\zeta^{2}\right)^{2}\right) \partial_{v}+\frac{1}{2} \zeta^{2} \partial_{u}+v(\zeta \partial) \\
& l(D)=2 v \partial_{v}+(\zeta \partial)
\end{aligned}
$$

The functions $C_{i}$ satisfy the equation (2.6) which for the $I$ and $I I$ cases can be rewritten as follows:

$$
C_{i}^{\prime \prime}+F(u) C_{i}=0 .
$$

The function $A$ is relevant only for the $I$ and $I I$ cases and it satisfies the following third-order differential equation:

$$
A^{\prime \prime \prime}+4 F(u) A^{\prime}+2 F^{\prime}(u) A=0 .
$$

Note that for the I case the isometry element $P_{u}$ is included into $T_{A}$.

Now let $y_{\alpha}, \alpha=1,2$, and $Y_{a}, a=1,2,3$, be independent solutions of the equation

$$
y^{\prime \prime}+F(u) y=0
$$

and (3.4), respectively. Consider the following brackets: 


$$
\begin{aligned}
& \left\{Y_{a}, y_{\beta}\right\} \equiv Y_{a} y_{\beta}^{\prime}-\frac{1}{2} y_{\beta} Y_{a}^{\prime}, \\
& \left\{y_{\alpha}, y_{\beta}\right\} \equiv y_{\alpha} y_{\beta}, \\
& \left\{Y_{a}, Y_{b}\right\} \equiv Y_{a} Y_{b}^{\prime}-Y_{b} Y_{a}^{\prime} .
\end{aligned}
$$

It is easy to prove that $\left\{Y_{a}, y_{\beta}\right\}$ and $\left\{y_{\alpha}, y_{\beta}\right\},\left\{Y_{a}, Y_{b}\right\}$ satisfy the equations (3.5) and (3.4), respectively. In other words, the following decompositions hold true:

$$
\begin{aligned}
& \left\{Y_{a}, y_{\beta}\right\}=f_{a \beta}^{\alpha} y_{\alpha}, \\
& \left\{y_{\alpha}, y_{\beta}\right\}=f_{\alpha \beta}^{a} Y_{a}, \\
& \left\{Y_{a}, Y_{b}\right\}=f_{a b}^{c} Y_{c} .
\end{aligned}
$$

An interesting fact is that the $y_{\alpha}$ and $Y_{a}$ equipped with commutation relations (3.7) constitute the superalgebra $\operatorname{osp}(2,1)$ (for proof see Appendix B). Now we are able to write down the commutation relations of our algebra:

$$
\begin{gathered}
{\left[D, P_{C}\right]=-P_{C}, \quad\left[D, P_{v}\right]=-2 P_{v}, \quad\left[D, K_{C}\right]=K_{C}, \quad\left[D, K_{u}\right]=2 K_{u},} \\
{\left[P_{C}, P_{B}\right]=W\left(C_{i}, B_{i}\right) P_{v}, \quad\left[K_{C}, K_{B}\right]=W\left(C_{i}, B_{i}\right) K_{u},} \\
{\left[T_{Y_{1}}, T_{Y_{2}}\right]=T_{\left\{Y_{1}, Y_{2}\right\}},} \\
{\left[T_{Y}, P_{C}\right]=P_{\{Y, C\}},} \\
{\left[T_{Y}, K_{C}\right]=K_{\{Y, C\}},} \\
{\left[K_{u}, P_{C}\right]=-K_{C},} \\
{\left[P_{v}, K_{C}\right]=P_{C},} \\
{\left[P_{C}, K_{B}\right]=T_{\left\{C_{i}, B_{i}\right\}}+W\left(C_{i}, B_{j}\right)\left(\frac{1}{2} \delta_{i j} D+J_{i j}\right),}
\end{gathered}
$$

where we introduce

$$
W\left(C_{i}, B_{j}\right) \equiv C_{i} B_{j}^{\prime}-B_{j} C_{i}^{\prime} .
$$

Note that due to eq.(3.3), the $W$ does not depend on $u$.

Now we are going to prove that the algebra of commutators of (3.9)-(3.15) is isomorphic to that of the usual conformal so $(4,2)$ algebra. To do that we prefer to use the 
'tensor' form of the algebra under consideration. First of all, let us write the general solution to (3.3) as follows:

$$
C_{i}=C_{0 i}^{+} y^{+}+C_{0 i}^{-} y^{-}, \quad y^{ \pm}=\left(y_{1} \pm \mathrm{i} y_{2}\right) / \sqrt{W},
$$

where $C_{0 i}^{ \pm}$are some constants while $y_{1}$ and $y_{2}$ are real-valued solutions to (3.5). For definiteness we consider the $W>0$ case. Note that three independent solutions to (3.4) can be written in terms of $y^{ \pm}$as follows:

$$
Y^{0}=y^{+} y^{-}, \quad Y^{ \pm \pm}=\left(y^{ \pm}\right)^{2} .
$$

Then we introduce desired generators

$$
\begin{array}{cc}
P_{i}^{ \pm}=\frac{\partial}{\partial C_{0 i}^{ \pm}} P_{C}, & K_{i}^{ \pm}=\frac{\partial}{\partial C_{0 i}^{ \pm}} K_{C}, \\
P_{u} \equiv T_{Y^{0}}, & J^{ \pm \pm} \equiv T_{Y^{ \pm \pm}} .
\end{array}
$$

Commutation relations between these elements can be readily derived from those of (3.9. 3.15). The elements $\left\{D, P_{v}, K_{u}\right\},\left\{P_{u}, J^{ \pm \pm}\right\}$, and $J_{i j}$ form three commutative subalgebras $K \equiv s o(2,1) \otimes s o(2,1) \otimes s o(2)$, respectively:

$$
\begin{gathered}
{\left[D, P_{v}\right]=-2 P_{v}, \quad\left[D, K_{u}\right]=2 K_{u}, \quad\left[P_{v}, K_{u}\right]=D} \\
{\left[J^{ \pm \pm}, P_{u}\right]= \pm 2 \mathrm{i} J^{ \pm \pm}, \quad\left[J^{++}, J^{--}\right]=4 \mathrm{i} P_{u} .}
\end{gathered}
$$

All remainder elements, which are $V \equiv P_{i}^{ \pm}, K_{i}^{ \pm}$, transform in their representations $[V, K] \in V:$

$$
\begin{gathered}
{\left[D, P_{i}^{ \pm}\right]=-P_{i}^{ \pm}, \quad\left[D, K_{i}^{ \pm}\right]=K_{i}^{ \pm},} \\
{\left[P_{i}^{ \pm}, K_{u}\right]=K_{i}^{ \pm}, \quad\left[K_{i}^{ \pm}, P_{v}\right]=-P_{i}^{ \pm},} \\
{\left[P_{i}^{ \pm}, P_{u}\right]= \pm \mathrm{i} P_{i}^{ \pm}, \quad\left[K_{i}^{ \pm}, P_{u}\right]= \pm \mathrm{i} K_{i}^{ \pm},} \\
{\left[J^{ \pm \pm}, P_{i}^{\mp}\right]= \pm 2 \mathrm{i} P_{i}^{ \pm}, \quad\left[J^{ \pm \pm}, K_{i}^{\mp}\right]= \pm 2 \mathrm{i} K_{i}^{ \pm},} \\
{\left[P_{i}^{ \pm}, J_{j k}\right]=\delta_{i j} P_{k}^{ \pm}-\delta_{i k} P_{j}^{ \pm}, \quad\left[K_{i}^{ \pm}, J_{j k}\right]=\delta_{i j} K_{k}^{ \pm}-\delta_{i k} K_{j}^{ \pm},}
\end{gathered}
$$

and satisfy the following commutation relations between each other $[V, V] \in K$ :

$$
\begin{gathered}
{\left[P_{i}^{+}, P_{j}^{-}\right]=2 \mathrm{i} \delta_{i j} P_{v}, \quad\left[K_{i}^{+}, K_{j}^{-}\right]=2 \mathrm{i} \delta_{i j} K_{u}} \\
{\left[P_{i}^{ \pm}, K_{j}^{\mp}\right]=\delta_{i j} P_{u} \pm \mathrm{i}\left(\delta_{i j} D+2 J_{i j}\right)} \\
{\left[P_{i}^{ \pm}, K_{j}^{ \pm}\right]=\delta_{i j} J^{ \pm \pm} .}
\end{gathered}
$$


In other words, the algebra under consideration has the Cartan-like decomposition

$$
G=V \boxplus K .
$$

Before formulating our statement, let us decompose $P_{i}^{ \pm}$and $K_{i}^{ \pm}$into real pieces

$$
\begin{gathered}
P_{i}^{ \pm}=P_{i} \mp \mathrm{i} J_{v i}, \\
K_{i}^{ \pm}= \pm \mathrm{i} K_{i}+J_{u i} .
\end{gathered}
$$

Now we are in a position to show explicitly an isomorphism between the algebra above and the elements of conformal so $(4,2)$ algebra taken in the Lorentz basis. Our statement is that the generators defined by

$$
\begin{gathered}
\mathbf{P}_{u}=\frac{1}{2} P_{u}+\frac{1}{4}\left(J^{++}+J^{--}\right), \\
\mathbf{K}_{v}=\frac{1}{2} P_{u}-\frac{1}{4}\left(J^{++}+J^{--}\right), \\
\mathbf{D}=\frac{1}{2} D+\frac{\mathrm{i}}{4}\left(J^{++}-J^{--}\right), \\
\mathbf{J}_{u v}=\frac{1}{2} D-\frac{\mathrm{i}}{4}\left(J^{++}-J^{--}\right), \\
\mathbf{P}_{v}=P_{v}, \quad \mathbf{P}_{i}=P_{i}, \quad \mathbf{K}_{u}=K_{u}, \quad \mathbf{K}_{i}=K_{i}, \\
\mathbf{J}_{v i}=J_{v i}, \quad \mathbf{J}_{u i}=J_{u i}, \quad \mathbf{J}_{i j}=J_{i j},
\end{gathered}
$$

satisfy the familiar commutation relations of the $s o(4,2)$ algebra

$$
\begin{array}{rlrl}
{\left[\mathbf{J}_{\mu \nu}, \mathbf{J}_{\rho \sigma}\right]} & =\eta_{\mu \rho} \mathbf{J}_{\sigma \nu}+\ldots, & & {\left[\mathbf{D}, \mathbf{P}_{\mu}\right]=-\mathbf{P}_{\mu},} \\
{\left[\mathbf{P}_{\mu}, \mathbf{J}_{\rho \sigma}\right]=\eta_{\mu \rho} \mathbf{P}_{\sigma}+\ldots,} & {\left[\mathbf{D}, \mathbf{K}_{\mu}\right]} & =\mathbf{K}_{\mu}, \\
{\left[\mathbf{K}_{\mu}, \mathbf{J}_{\rho \sigma}\right]} & =\eta_{\mu \rho} \mathbf{K}_{\sigma}+\ldots, & & {\left[\mathbf{D}, \mathbf{J}_{\mu \nu}\right]=0,} \\
{\left[\mathbf{P}_{\mu}, \mathbf{K}_{\nu}\right]=\eta_{\mu \nu} \mathbf{D}-\mathbf{J}_{\mu \nu}, \quad\left[\mathbf{P}_{\mu}, \mathbf{P}_{\nu}\right]=0,} & {\left[\mathbf{K}_{\mu}, \mathbf{K}_{\nu}\right]=0,}
\end{array}
$$

where nonvanishing elements of $\eta_{\mu \nu}$ are given by $\eta_{u v}=\eta_{v u}=1$ and $\eta_{i j}=-\delta_{i j}$. Making use of (3.16-3.27), this statement can be easily proved. For future reference let us describe how we link generators above with those taken in the six dimensional frame. We introduce $J_{A B}, A, B=(\mu, 5,6)$, as

$$
\begin{aligned}
& \mathbf{P}_{\mu}=\left(J_{5 \mu}+J_{6 \mu}\right) / \sqrt{2}, \quad \mathbf{K}_{\mu}=\left(J_{5 \mu}-J_{6 \mu}\right) / \sqrt{2}, \\
& \mathbf{J}_{\mu \nu}=J_{\mu \nu}, \quad \mathbf{D}=J_{56}
\end{aligned}
$$


which satisfy the well-known commutation relations

$$
\left[J_{A B}, J_{C D}\right]=\eta_{A C} J_{D B}+\ldots,
$$

where $\eta_{u v}=\eta_{v u}=\eta_{55}=-\eta_{11}=-\eta_{22}=-\eta_{66}=1$.

Now we would like to write down the expressions for the spin parts of the conformal algebra elements. The procedure of evaluation is the same that we used for the spin parts of isometry algebra (see Appendix A). The result is given by

$$
\begin{aligned}
& s\left(T_{A}\right)=\frac{1}{2} A^{\prime \prime} \zeta_{i} M_{V i}+\frac{1}{2} A^{\prime}\left(\Delta+2 M_{V U}\right), \\
& s\left(K_{C}\right)=\left(v C_{i}^{\prime}-F(u) \zeta_{i}(\zeta C)\right) M_{V i}+C_{i} M_{U i}+\left(\zeta C^{\prime}\right)\left(\Delta+M_{V U}\right)+\zeta_{i} C_{j}^{\prime} M_{i j}, \\
& s\left(K_{u}\right)=-\frac{1}{2} F(u) \zeta_{i} \zeta^{2} M_{V i}+\zeta_{i} M_{U i}+v \Delta, \\
& s(D)=\Delta .
\end{aligned}
$$

Let us comment on the expressions above. As is seen from (2.3)-(2.5) and (3.2) the little algebra which leaves the point $x=0$ invariant is given by eleven elements that are six homogeneous Lorentz transformations $M_{A B}$, dilatation $\Delta$, and special conformal transformations $\kappa_{A}=\left(\kappa_{U}, \kappa_{V}, \kappa_{i}\right)$. The expressions (3.30) include only $M_{A B}$ and $\Delta$ parts. As usual, the $\kappa_{A}$ part of the conformal algebra can be obtained by considering the homogeneous so $(4,2)$ transformations. The result is given by

$$
\begin{aligned}
& k\left(T_{A}\right)=\frac{1}{2} A^{\prime \prime} \kappa_{V}, \\
& k\left(K_{C}\right)=-F(u)(C \zeta) \kappa_{V}-\left(C^{\prime} \kappa\right), \\
& k\left(K_{u}\right)=\kappa_{U}-\frac{1}{2} F(u) \zeta^{2} \kappa_{V},
\end{aligned}
$$

where $\kappa_{A}$ transforms in vector representation of the $s o(3,1)$ algebra:

$$
\left[\kappa_{A}, M_{B C}\right]=\eta_{A B} \kappa_{C}-\eta_{A C} \kappa_{B} .
$$

Note that $\left[\Delta, \kappa_{A}\right]=0$, while the commutator $\left[\Delta, M_{A B}\right]$ can be read from (A.1), (A.2), and (2.11). Finally, the complete expressions for conformal generators read as

$$
G=l(G)+s(G)+k(G) .
$$

\section{Massless Fields}

\section{A. Gauge invariant equations of motion}

With conformal algebra at our hands we are ready to provide a constructive definition of massless fields living in plane wave geometry. Notice from now on we restrict ourselves 
to the I case because for this case only there exists a conserved energy Killing vector. By analogy with Minkowski and de Sitter spaces we define plane wave geometry massless fields as those whose wave equations are conformal invariant. Now let us construct relevant wave equations.

First of all let us collect results concerning the manifest expressions for isometry algebra elements. From now on to simplify our expressions we shall put $F=1$. Then making use (2.4) and (3.3) one gets

$$
\begin{gathered}
P_{u}=\partial_{u}, \quad P_{v}=\partial_{v}, \\
P_{i}^{ \pm}=e^{\mp \mathrm{i} u}\left(\partial_{i} \mp \mathrm{i} \zeta_{i} \partial_{v} \mp \mathrm{i} M_{V i}\right), \\
J_{i j}=-\zeta_{i} \partial_{j}+\zeta_{j} \partial_{i}+M_{i j} .
\end{gathered}
$$

From the commutation relations which satisfy the generators above (see 3.22 and 3.23 ) it is readily seen that the algebra under consideration has the following Levy decomposition:

$$
G=N \boxplus S
$$

where $S$ is a 'semisimple' algebra $s o(2)$ spanned by $J_{i j}$ while $N$ is a maximal solvable ideal (radical) spanned by $P_{u}, P_{v}, P_{i}^{ \pm}$. Note that the $N$ is nothing but a six dimensional Heisenberg algebra $H_{6}$. This fact can be readily seen from (3.20) and (3.23). As is known, the Killing metric for the $H_{6}$ is degenerate. By analogy with the Poincare algebra one might replace it by a certain invariant symmetric nondegenerate bilinear form. In turns out that there exist two forms: the first form leads to the following second-order operator,

$$
Q=2 P_{u} P_{v}-\frac{1}{2} P_{i}^{+} P_{i}^{-}-\frac{1}{2} P_{i}^{-} P_{i}^{+},
$$

and it is an analog of $p^{2}$, while the second form leads to

$$
Q_{\lambda}=\epsilon_{i j} J_{i j} P_{v}+\mathrm{i} \epsilon_{i j} P_{i}^{+} P_{j}^{-},
$$

which is the helicity operator [for definition of $\epsilon_{i j}$ see (4.47)].

To clarify the algebra structure from the point of view of space-time transformations, let we rewrite our algebra in terms of $P_{i}$ and $J_{v i}$ [see (3.26)]:

$$
\begin{gathered}
P_{i}=\cos u \partial_{i}-\sin u \zeta_{i} \partial_{v}-\sin u M_{V i}, \\
J_{v i}=\sin u \partial_{i}+\cos u \zeta_{i} \partial_{v}+\cos u M_{V i},
\end{gathered}
$$

which satisfy the commutation relations

$$
\begin{gathered}
{\left[P_{i}, J_{j k}\right]=-\delta_{i j} P_{k}+\delta_{i k} P_{j},} \\
{\left[J_{v i}, J_{j k}\right]=-\delta_{i j} J_{v k}+\delta_{i k} J_{v j},} \\
{\left[P_{u}, J_{v i}\right]=P_{i}, \quad\left[P_{i}, J_{v j}\right]=\delta_{i j} P_{v},}
\end{gathered}
$$




$$
\left[P_{u}, P_{i}\right]=-J_{v i}
$$

From the expressions (4.1), 4.6, and (4.7), it is clear that the generators

$$
P_{u}, \quad P_{v}, \quad P_{i}
$$

do not leave point $x^{\mu}=0$ invariant, while rotation generators (i.e., their orbital parts)

$$
J_{i j}, \quad J_{v i},
$$

leave the point $x^{\mu}=0$ invariant. In other words, the generators $P_{u}, P_{v}$, and $P_{i}$ can be interpreted as curved counterparts of translation elements of Poincare algebra while $J_{i j}$ and $J_{v i}$ are counterparts of the Lorentz subalgebra. In terms of these generators the first Casimir operator [see 4.4] reads as

$$
Q=2 P_{u} P_{v}-P_{i} P_{i}-J_{v i} J_{v i}
$$

Now we are ready to discuss two possible definitions of masslessness based on conformal algebra. The first definition is formulated as the following requirements on the wavefunction:

$$
[G, Q]|\Phi\rangle=0
$$

where $G$ are all of the elements of conformal algebra. The second definition actually is based on so-called $\operatorname{sim}(3,1)$ algebra, which is the isometry algebra combined with dilatation (see Ref. [13). Since elements of isometry algebra commutate with $Q$, the second definition amounts to the condition

$$
[D, Q]|\Phi\rangle=0
$$

which should, strictly speaking, be accompanied by certain things (see below). Note that for the case of Minkowski space-time the second definition describes all massless states provided by considerations of Poincare algebra representations. A surprising fact discovered in Ref.[14] is that the first definition describes all massless states only for the case of four dimensional $(d=4)$ Minkowski space-time. Namely, in Ref. [14 it was demonstrated that for the massless representations of Poincare algebra in $d>4$ the first definition leads to so-called degenerate representations which constitute only a subset of all massless states. This result for the case of massless representations of an anti-de Sitter algebra has been generalized in Ref. 115], i.e., it seems that this phenomena is an inherent feature of all massless fields irrespective of the manifolds were they propagate. Since we are considering four-dimensional space-time we expect that the first definition describes all massless representation, i.e., there are no restrictions on allowed value of spin. Below, among other things, we will demonstrate that this is indeed the case.

Let us start with the second definition. Because of the relation [see (3.16), (3.18), and (3.26)]

$$
[D, Q]=-2 Q
$$


we conclude that wave equation for massless fields must read

$$
Q|\Phi\rangle=0
$$

As is known in four-dimensional space-time all massless fields can be described by means of totally symmetric tensor fields, i.e., in terms of potentials. In this case to provide a complete description of massless fields the equation of motion (4.11) must be supplemented by corresponding gauge transformation and subsidiary conditions. Now we are going to establish such conditions and gauge transformation.

First of all, due to (4.1) and (4.2) one has the following representation for the operator $Q$ :

$$
Q=2 e_{U} e_{V}-e_{i} e_{i}-2 \zeta_{i} M_{V i} e_{V}-M_{V i} M_{V i},
$$

where the dual forms $e_{A}$ are given by (2.9). Then we introduce the gauge transformation

$$
\delta|\Phi\rangle=R|\alpha\rangle
$$

and postulate the following subsidiary conditions:

$$
\begin{array}{lc}
\bar{R}|\Phi\rangle=0 \quad \text { (divergencelessness) }, \\
\bar{a}^{A} \bar{a}_{A}|\Phi\rangle=0 \quad \text { (tracelessness) } \\
\bar{a}_{V}^{2}|\Phi\rangle=0,
\end{array}
$$

where the operators $R$ and $\bar{R}$ are defined by

$$
\begin{gathered}
R \equiv a^{A} D_{A}, \quad \bar{R} \equiv \bar{a}^{A} D_{A}, \\
D_{A}=e_{A}^{\mu} D_{\mu},
\end{gathered}
$$

and $D_{\mu}$ is a Lorentz covariant derivative

$$
D_{\mu} \equiv \partial_{\mu}+\frac{1}{2} \omega_{\mu}^{A B} M_{A B}
$$

From (2.8) it is readily seen that $D_{\mu}=\partial_{\mu}-\delta_{\mu}^{u} \zeta_{i} M_{V i}$ where a relevant representation for $M_{A B}$ is given by (A.1). The $|\alpha\rangle$ in (4.13) is a parameter of gauge transformation. If the $|\Phi\rangle$ is a degree- $s$ monomial in the oscillator $a_{A}$ then the $|\alpha\rangle$ is a degree- $(s-1)$ monomial in $a_{A}$. We impose on $|\alpha\rangle$ the following equation of motion:

$$
Q|\alpha\rangle=0
$$

and the subsidiary conditions

$$
\begin{array}{lc}
\bar{R}|\alpha\rangle=0 \quad \text { (divergencelessness) }, \\
\bar{a}^{A} \bar{a}_{A}|\alpha\rangle=0 \quad \text { (tracelessness) } \\
\bar{a}_{V}|\alpha\rangle=0 .
\end{array}
$$


Let us make a few comments on the gauge transformation and subsidiary conditions above. As to (4.14,4.18), (4.15), and (4.19) these are nothing but familiar conditions of divergencelessness and tracelessness respectively formulated on tangent space tensor fields. The conditions (4.16) and (4.20) are less familiar. These can be rewritten in the following covariant fashion:

$$
R_{A B} \bar{a}^{A} \bar{a}^{B}|\Phi\rangle=0, \quad R_{A B} \bar{a}^{A} D^{B}|\alpha\rangle=0,
$$

where $R_{A B}=2 \delta_{A}^{U} \delta_{B}^{U}$ is the Ricci tensor in tangent space. Note that the second subsidiary condition from (4.21) is equivalent to (4.20) provided $\partial_{v}|\alpha\rangle \neq 0$.

Making use of (4.17)-(4.20) and commutation relations

$$
\begin{gathered}
{[R, \bar{R}]=Q+2 M_{V i} M_{V i},} \\
{[R, Q]=0, \quad[\bar{R}, Q]=0,} \\
{\left[\bar{a}^{A} \bar{a}_{A}, R\right]=-2 \bar{R}, \quad\left[a^{A} a_{A}, \bar{R}\right]=2 R,}
\end{gathered}
$$

one can make sure that gauge transformation (4.13) respects the equation (4.11) and subsidiary conditions (4.14)-(4.16).

Now we are going to prove the following main result of this subsection.

Proposition: Let the gauge parameter $|\alpha\rangle$ satisfy (4.17)-(4.20). Then the equation (4.11) and subsidiary conditions (4.14)-(4.16) supplemented by gauge transformation (4.13) describe the $\left|\Phi_{t r}^{(0)}\right\rangle$ which (1) depends only on transversal oscillator $a_{i}$; and (2) satisfies the equation of motion

$$
\square_{0}\left|\Phi_{t r}^{(0)}\right\rangle=0
$$

and tracelessness condition in transversal directions

$$
\bar{a}_{i} \bar{a}_{i}\left|\Phi_{t r}^{(0)}\right\rangle=0
$$

where in (4.22) $\square_{0}=2 e_{U} e_{V}-e_{i} e_{i}$ is the second-order operator for massless scalar field

$$
\square_{0}=2 \partial_{u} \partial_{v}-\zeta^{2} \partial_{v}^{2}-\partial^{2}
$$

Note that (4.23) tells us that actually there are only two polarization degrees of freedom, while (4.22) expresses the fact that these physical degrees of freedom satisfy the same equation of motion as the spinless (scalar) field. In these respects there is analogy with massless fields in Minkowski space-time.

Proof: From (4.16) and (4.20) it follows

$$
\begin{gathered}
|\Phi\rangle=\left|\Phi^{(0)}\right\rangle+a_{U}\left|\Phi^{(1)}\right\rangle, \\
|\alpha\rangle=\left|\alpha^{(0)}\right\rangle,
\end{gathered}
$$

where generating functions $\left|\Phi^{(0)}\right\rangle,\left|\Phi^{(1)}\right\rangle$, and $\left|\alpha^{(0)}\right\rangle$ are independent of $a_{U}$. Now substituting (4.25) into (4.11) we get for $\left|\Phi^{(0)}\right\rangle$ and $\left|\Phi^{(1)}\right\rangle$ the following equations of motion: 


$$
\begin{gathered}
Q\left|\Phi^{(0)}\right\rangle+2\left(\zeta_{i} a_{i} e_{V}-a_{V}+a_{i} M_{V i}\right)\left|\Phi^{(1)}\right\rangle=0, \\
Q\left|\Phi^{(1)}\right\rangle=0 .
\end{gathered}
$$

By substituting (4.25) into (4.13) we get the gauge transformations

$$
\begin{gathered}
\delta\left|\Phi^{(0)}\right\rangle=\left(a_{V}\left(e_{U}-\zeta_{i} M_{V i}\right)-a_{i} e_{i}\right)\left|\alpha^{(0)}\right\rangle, \\
\delta\left|\Phi^{(1)}\right\rangle=e_{V}\left|\alpha^{(0)}\right\rangle
\end{gathered}
$$

and by substituting (4.25) into (4.14) and (4.15) we obtain the constraints

$$
\begin{gathered}
\left(\bar{a}_{U} e_{V}-\bar{a}_{i} e_{i}\right)\left|\Phi^{(0)}\right\rangle-\left(e_{U}-\zeta_{i} M_{V i}\right)\left|\Phi^{(1)}\right\rangle=0, \\
\left(\bar{a}_{U} e_{V}-\bar{a}_{i} e_{i}\right)\left|\Phi^{(1)}\right\rangle=0, \\
\bar{a}_{i} \bar{a}_{i}\left|\Phi^{(0)}\right\rangle+2 \bar{a}_{U}\left|\Phi^{(1)}\right\rangle=0, \\
\bar{a}_{i} \bar{a}_{i}\left|\Phi^{(1)}\right\rangle=0 .
\end{gathered}
$$

By using (4.17)-(4.19) and (4.26) we repeat the procedure above for $\left|\alpha^{(0)}\right\rangle$ and get the following equation of motion and constraints:

$$
\begin{gathered}
Q\left|\alpha^{(0)}\right\rangle=0, \\
\left(\bar{a}_{U} e_{V}-\bar{a}_{i} e_{i}\right)\left|\alpha^{(0)}\right\rangle=0, \\
\bar{a}_{i} \bar{a}_{i}\left|\alpha^{(0)}\right\rangle=0 .
\end{gathered}
$$

Now taking into account that $\left|\Phi^{(1)}\right\rangle$ and $\left|\alpha^{(0)}\right\rangle$ satisfy the same equations of motion [see (4.28 and (4.35)) as well as the same constraints [see (4.32), (4.34) (4.36), and (4.37)] we can, due to (4.30), impose the following light cone like gauge:

$$
\left|\Phi^{(1)}\right\rangle=0 \text {. }
$$

By substituting the gauge (4.38) into (4.33) and (4.31) we get

$$
\begin{gathered}
\bar{a}_{i} \bar{a}_{i}\left|\Phi^{(0)}\right\rangle=0, \\
\left(\bar{a}_{U} e_{V}-\bar{a}_{i} e_{i}\right)\left|\Phi^{(0)}\right\rangle=0 .
\end{gathered}
$$

Constraint (4.39) tells us that $\left|\Phi^{(0)}\right\rangle$ is a traceless tensor with respect to transversal indices $i_{1}, \ldots, i_{s}$. The solution to (4.40) can be written as 


$$
\left|\Phi^{(0)}\right\rangle=\exp \left(-\frac{e_{i}}{e_{V}} \bar{a}_{i} a_{V}\right)\left|\Phi_{t r}^{(0)}\right\rangle,
$$

where subscript (tr) indicates the fact that $\left|\Phi_{t r}^{(0)}\right\rangle$ depends only on the transversal oscillator $a_{i}$. By substituting (4.41) into (4.39) we get (4.23), while by substituting (4.41) and (4.38) into (4.27) we arrive at (4.22). The proposition is proved.

In conclusion of this subsection let us note that helicity operator $Q_{\lambda}$ from (4.5) is realized on $\left|\Phi_{t r}^{(0)}\right\rangle$ as follows:

$$
Q_{\lambda}\left|\Phi_{t r}^{(0)}\right\rangle=\epsilon_{i j} M_{i j} \partial_{v}\left|\Phi_{t r}^{(0)}\right\rangle,
$$

and as is easily seen takes two values equal to $\pm \lambda p_{v}$.

\section{B. Light cone representation for conformal algebra}

In this subsection by making use of the first definition we shall derive the expressions for elements of conformal algebra acting as differential operators on the solution of (4.22) and defined on a surface of initial conditions $u=0$. In other words we are going to investigate the complete set of the equations which follows from (4.9). As is well known, the complete set of equations is obtained by performing double conformal boost, i.e., the complete set of equations is given by $[G, Q]|\Phi\rangle=0$ as well as $[G,[G, Q]]|\Phi\rangle=0$. Note that the set of equations in question can be rewritten in a form which is manifestly invariant with respect to conformal algebra:

$$
T_{A B}=0,
$$

where

$$
T_{A B}=\left\{J_{A C}, J_{C B}\right\}+\frac{1}{3} \eta_{A B} J_{C D} J_{C D},
$$

where $\{a, b\} \equiv a b+b a$. These equations we analyze on the surface of initial conditions $u=0$ and make use of light cone like gauge

$$
M_{V i}=0 .
$$

Note that we prefer to pass from wavefunction $\Phi\left(u, v, \zeta_{i}\right)$ to its Fourier modes with respect to $v$, i.e., in what follows we shall replace operator $\partial_{v}$ by $-\mathrm{i} p_{v}$.

Again one has the equation of motion (4.11), making use of which as well as (4.12) and (4.43) we get on-mass shell condition

$$
\left.\left(\partial_{u} \Phi\right)\right|_{u=0}=\frac{\mathrm{i}}{2 p_{v}}\left(\partial^{2}-p_{v}^{2} \zeta^{2}\right) \Phi\left(p_{v}, \zeta\right) .
$$

Now making use of (3.28) and (3.31) we are able to learn restrictions which impose (4.42) on $M_{A B}, \Delta$, and $\kappa_{A}$. Because the analysis of eqs. (4.42) is straightforward but very tedious, we outline the procedure of solution and present the results.

From $T_{+V}=0$ one gets 


$$
M_{U V}=\frac{1}{2}(1-\Delta),
$$

while from $T_{+i}=0$ we learn

$$
M_{U i}=-\frac{\mathrm{i}}{p_{v}} M_{i j} \partial_{j}+\frac{\mathrm{i}}{p_{v}} \partial_{i} .
$$

Making use of these results we derive from equations $T_{--}=0, T_{-i}=0$, and $T_{+-}=0$,

$$
\kappa_{V}=0, \quad \kappa_{i}=0, \quad \Delta=1,
$$

respectively, while from $T_{+i}=0$ one gets

$$
\kappa_{U}=\frac{\mathrm{i}}{2 p_{v}} M^{2}, \quad M^{2} \equiv M_{i j} M_{i j} .
$$

Now collecting all results above we get the following light cone representation for the generators of conformal group acting on the wavefunction of plane wave geometry massless fields $\Phi\left(p_{v}, \zeta\right)$ :

$$
\begin{aligned}
& ( \pm) \mathbf{P}_{u}=\frac{\mathrm{i}}{2 p_{v}} \partial^{2}, \quad( \pm) \mathbf{P}_{v}=-\mathrm{i} p_{v}, \quad( \pm) \mathbf{P}_{i}=\partial_{i}, \\
& \mathbf{J}_{i j}=-\zeta_{i} \partial_{j}+\zeta_{j} \partial_{i}+M_{i j}, \\
& \mathbf{J}_{v i}=-\mathrm{i} \zeta_{i} p_{v} \\
& \mathbf{J}_{u v}=-\mathrm{i} v p_{v}, \\
& \mathbf{J}_{u i}=v \partial_{i}+\frac{\mathrm{i}}{2 p_{v}} \zeta_{i} \partial^{2}-\frac{\mathrm{i}}{p_{v}} M_{i j} \partial_{j}, \\
& ( \pm) \mathbf{K}_{u}=v \mathbf{D}+\frac{\mathrm{i}}{4 p_{v}} \zeta^{2} \partial^{2}-\frac{\mathrm{i}}{p_{v}} \zeta_{i} M_{i j} \partial_{j}+\frac{\mathrm{i}}{2 p_{v}} M^{2} \\
& ( \pm) \mathbf{K}_{v}=-\frac{\mathrm{i}}{2} \zeta^{2} p_{v}, \\
& ( \pm) \mathbf{K}_{i}=-\zeta_{i} \mathbf{D}+\frac{1}{2} \zeta^{2} \partial_{i}+M_{i j} \zeta_{j}, \\
& \mathbf{D}=-\mathrm{i} v p_{v}+\zeta_{j} \partial_{j}+1 .
\end{aligned}
$$

Note that while writing the final representation (4.45) we have assigned + and - to particles and antiparticles, respectively, and have put $p_{v}>0$ for both particles and antiparticles. It should be emphasized that all analysis above is sensitive to space-time dimension because from the equation $T_{i j}=0$ it follows

$$
\left\{M_{i k}, M_{j k}\right\}=\delta_{i j} M^{2} .
$$


It is the equation (4.46) that leads to degenerate representation in higher space time dimensions $d>4$ (see Refs. [14] and [15]). Fortunately, in four dimensions, due to relations

$$
M_{i j}=\mathrm{i} \epsilon_{i j} \lambda, \quad \epsilon_{i j}=-\epsilon_{j i}, \quad \epsilon_{12}=1,
$$

the equation (4.46) does not impose of any restriction on helicity value $\lambda$.

\section{Energy lowest value}

In this subsection we are going to demonstrate one interesting phenomena of massless fields in plane wave geometry. Namely we would like to show explicitly that (1) energy operator i $P_{u}\left(\mathrm{i} P_{u} \Phi=\mathrm{i} \partial_{u} \Phi\right)$ is bounded from below (above) for particles (antiparticles) and (2) it takes only discrete values.

As we have shown physical degrees of freedom of massless arbitrary spin fields satisfy the equation

$$
\left(2 \partial_{u} \partial_{v}-\zeta^{2} \partial_{v}^{2}-\partial_{i}^{2}\right) \Phi=0
$$

First of all we introduce a radial variable $\eta$ and angle $\phi$ by

$$
\zeta=\frac{\sqrt{\eta}}{2 \sqrt{\left|p_{v}\right|}} e^{\mathrm{i} \phi} .
$$

Note that in (4.49) we use the complex variable $\zeta$ given by (2.2). Then we pass to Fouirer modes with respect to $u, v$, and $\phi$, i.e., we put

$$
\partial_{u} \Phi=-\mathrm{i} p_{u} \Phi, \quad \partial_{v} \Phi=-\mathrm{i} p_{v} \Phi, \quad \partial_{\phi} \Phi=\mathrm{i} k \Phi,
$$

$k=0, \pm 1, \pm 2 \ldots$, and use the substitution

$$
\Phi=e^{-\eta / 2} \eta^{|k| / 2} \omega(\eta) .
$$

As a result, one has a differential equation for degenerate hypergeometric function

$$
\eta \omega^{\prime \prime}+(\gamma-\eta) \omega^{\prime}-\alpha \omega=0
$$

where in (4.51) prime indicates partial derivative with respect to $\eta$ while $\gamma$ and $\alpha$ are given by

$$
\gamma=|k|+1, \quad \alpha=\frac{1}{2}\left(-p_{u} \operatorname{sign} p_{v}+|k|+1\right),
$$

where $\operatorname{sign} p_{v}=1(-1)$ for $p_{v}>0\left(p_{v}<0\right)$. Solution to (4.51) is $\omega=F(\alpha, \gamma, \eta)$. It is straightforward now verify (see Ref. [16]) that in order for $\Phi$ [see 4.50] to be squareintegrable one should take $\alpha=-n$, where $n=0,1, \ldots$ For such $\alpha$ the $\omega$ is nothing but the generalized Laggere polynomial $\omega=L_{n}^{(|k|)}(\eta)$. Now taking into account (4.52), we arrive at the following spectrum for $p_{u}$ :

$$
p_{u}=\operatorname{sign} p_{v}(2 n+|k|+1) .
$$


Note that with to respect the dimensionfull parameter $F$ we should multiply the rhs of (4.53) by $\sqrt{F}$. The $p_{v}>0$ corresponds to particles while $p_{v}<0$ corresponds to antiparticles. Note that in contrast to particles in Minkowski space-time, the $p_{u}$ does not reach zero value:

$$
\begin{array}{ll}
p_{v}>0 ; & \min p_{u}=1, \\
p_{v}<0 ; & \max p_{u}=-1 .
\end{array}
$$

In this respect plane wave geometry massless particles are similar to anti-de Sitter massless particles (see Refs. 177- 19]). The analogy to de-Sitter particles can be drawn further. First of all there exists vacuum state $|v a c\rangle$ defined by $P_{i}^{-}|v a c\rangle=0$. Second, level $n$ states can be obtained by operating with $P_{i}^{+}$on the vacuum: $P_{i_{1}}^{+} \ldots P_{i_{n}}^{+}|v a c\rangle$. Note that making use of equation $Q|v a c\rangle=0$ and (4.8) we immediately obtain the lowest energy value mentioned above.

\section{Ladder Representation}

In this section we would like to make contact with Ref.[20]. In Ref. 20] (see also Ref. 21]) it was demonstrated that a chain of massless representations of Poincare algebra consisting of all helicity states $\lambda=0, \pm 1 / 2, \pm 1, \ldots$ (where every state appears just once) can be embedded into a ladder representation of $u(2,2)$ algebra. We will demonstrate that the same chain of plane wave geometry massless states can also be embedded into the same ladder representation. As result we will able to show explicitly the manner in which Minkowski and plane wave geometry massless fields are related to each other.

A second long-term motivation of our interest in ladder representation comes from recent progress in the problem of massless higher spin fields dynamics ([22]). Namely, for the case of anti-de Sitter space-time in Ref.([22]) it was demonstrated that to construct self-consistent interaction of massless higher spin fields it is necessary, among other things, to introduce the chain anti-de Sitter massless fields which consists of every spin just once. Due to that, it is strongly believed that ladder representations can get interesting applications in future studies of massless higher spin fields interactions and deserve further investigation.

Let us briefly describe how the ladder representation is constructed (for details see Refs. 20] and [23]). Define the operator-valued four-component spinor $\varphi=\left(\varphi^{\alpha}\right)$ and $\tilde{\varphi}=\varphi^{*} \beta=\left(\tilde{\varphi}_{\alpha}\right), \alpha=1,2,3,4$, and impose the commutation relations

$$
\left[\varphi^{\alpha}, \tilde{\varphi}_{\beta}\right]=\delta_{\beta}^{\alpha}
$$

where

$$
\beta= \pm \gamma_{0}
$$

and $\gamma_{\mu}$ are Dirac $\gamma$-matrices subjected as usual to relation

$$
\left\{\gamma_{\mu}, \gamma_{\nu}\right\}=2 \eta_{\mu \nu}, \quad \eta_{\mu \nu}=\operatorname{diag}(1,-1,-1,-1)
$$


The signs + and - in (5.2) and below correspond to representations which are associated with particle or antiparticle, respectively. Now the ladder representation of $u(2,2)$ is defined by

$$
J_{A B}=\tilde{\varphi} \Sigma_{A B} \varphi, \quad C_{1}=\tilde{\varphi} \varphi
$$

where

$$
\begin{gathered}
\Sigma_{A B}=\left\{\Sigma_{\mu \nu}, \Sigma_{5 \mu}, \Sigma_{6 \mu}, \Sigma_{56}\right\}, \\
\Sigma_{\mu \nu}=\frac{1}{4}\left[\gamma_{\mu}, \gamma_{\nu}\right], \quad \Sigma_{56}=\frac{\mathrm{i}}{2} \gamma_{5}, \\
\Sigma_{5 \mu}=-\frac{\mathrm{i}}{2} \gamma_{\mu}, \quad \Sigma_{6 \mu}=\frac{1}{2} \gamma_{\mu} \gamma_{5} .
\end{gathered}
$$

Note that $\Sigma_{A B}$ satisfy the same commutations relations as $J_{A B}$. We use the following representation for $\gamma$-matrices:

$$
\gamma_{\mu}=\left(\begin{array}{ll}
0 & \bar{\sigma}_{\mu} \\
\sigma_{\mu} & 0
\end{array}\right), \quad \sigma_{\mu}=\left(1, \sigma_{i}\right), \quad \bar{\sigma}_{\mu}=\left(1,-\sigma_{i}\right),
$$

where $\sigma_{i}, i=1,2,3$, are usual Pauli matrices. Note that $\sigma_{u, v}$ are given by $\sigma_{u}=\left(\sigma_{0}+\right.$ $\left.\sigma_{3}\right) / \sqrt{2}$ and $\sigma_{v}=\left(\sigma_{0}-\sigma_{3}\right) / \sqrt{2}$. The $\gamma_{5}$ is defined by $\gamma_{5}=\gamma_{0} \gamma_{1} \gamma_{2} \gamma_{3}$.

To carry out the reduction we should rewrite the elements of the $u(2,2)$ algebra as differential operators acting on functions of $p_{v}$ and $\zeta_{i}$. To do that let us consider the space of Hillbert space of finite norm functions where the norm is defined by

$$
(f, g)=\int d^{2} z_{1} d^{2} z_{2} \overline{f\left(z_{1}, z_{2}\right)} g\left(z_{1}, z_{2}\right) .
$$

Now we choose the following representation for operator-valued four-component spinor $\varphi$

$$
\varphi=\left(\begin{array}{c}
\lambda \\
\pm \tau
\end{array}\right), \quad \lambda=\left(\begin{array}{c}
\partial_{z_{1}} \\
\bar{z}_{2}
\end{array}\right), \quad \tau=\left(\begin{array}{c}
\bar{z}_{1} \\
\partial_{z_{2}}
\end{array}\right)
$$

where we introduce two-component spinors $\lambda=\left(\lambda^{a}\right)$ and $\tau=\left(\tau^{a}\right)$, a $=1,2$, which satisfy the commutation relations

$$
\left[\lambda^{a}, \tau_{b}^{*}\right]=\delta_{b}^{a}, \quad\left[\tau^{a}, \lambda_{b}^{*}\right]=\delta_{b}^{a} .
$$

Due to (5.3) we have the following involution rules $(\partial / \partial z)^{*}=-\partial / \partial z$ and $z^{*}=\bar{z}$, i.e.,

$$
\tilde{\varphi}=\left(\tau^{*}, \pm \lambda^{*}\right), \quad \lambda^{*}=\left(-\partial_{\bar{z}_{1}}, z_{2}\right), \quad \tau^{*}=\left(z_{1},-\partial_{\bar{z}_{2}}\right) .
$$

Making use of (3.29) and (5.4) we derive the following representation for elements of $u(2,2)$ algebra in Lorentz basis

$$
\begin{aligned}
& ( \pm) \mathbf{P}_{\mu}=-\frac{\mathrm{i}}{\sqrt{2}} \lambda^{*} \sigma_{\mu} \lambda, \quad( \pm) \mathbf{K}_{\mu}=-\frac{\mathrm{i}}{\sqrt{2}} \tau^{*} \bar{\sigma}_{\mu} \tau, \\
& \mathbf{J}_{\mu \nu}=\lambda^{*} \sigma_{\mu \nu} \tau+\tau^{*} \bar{\sigma}_{\mu \nu} \lambda, \quad \mathbf{D}=\frac{1}{2}\left(\tau^{*} \lambda-\lambda^{*} \tau\right)
\end{aligned}
$$




$$
C_{1}=\lambda^{*} \tau+\tau^{*} \lambda
$$

which is very convenient for practical calculations. Making use of this representation we get the following expressions for generators:

$$
\begin{aligned}
& ( \pm) \mathbf{P}_{u}=\mathrm{i} \partial_{z_{1}} \partial_{\bar{z}_{1}}, \quad( \pm) \mathbf{K}_{u}=\mathrm{i} \partial_{z_{2}} \partial_{\bar{z}_{2}}, \quad \mathbf{J}_{u \zeta}=-\partial_{z_{1}} \partial_{\bar{z}_{2}}, \\
& ( \pm) \mathbf{P}_{v}=-\mathrm{i} z_{2} \bar{z}_{2}, \quad( \pm) \mathbf{K}_{v}=-\mathrm{i} z_{1} \bar{z}_{1}, \quad \mathbf{J}_{u \bar{\zeta}}=\partial_{\bar{z}_{1}} \partial_{z_{2}}, \\
& ( \pm) \mathbf{P}_{\zeta}=-\mathrm{i} z_{2} \partial_{z_{1}}, \quad( \pm) \mathbf{K}_{\zeta}=-\mathrm{i} \bar{z}_{1} \partial_{\bar{z}_{2}}, \quad \mathbf{J}_{v \zeta}=-\bar{z}_{1} z_{2}, \\
& ( \pm) \mathbf{P}_{\bar{\zeta}}=\mathrm{i} \bar{z}_{2} \partial_{\bar{z}_{1}}, \quad( \pm) \mathbf{K}_{\bar{\zeta}}=\mathrm{i} z_{1} \partial_{z_{2}}, \quad \mathbf{J}_{v \bar{\zeta}}=z_{1} \bar{z}_{2}, \\
& \mathbf{J}_{u v}=-\frac{1}{2}\left(z_{1} \partial_{z_{1}}+z_{2} \partial_{z_{2}}+\partial_{\bar{z}_{1}} \bar{z}_{1}+\partial_{\bar{z}_{2}} \bar{z}_{2}\right) \text {, } \\
& \mathbf{D}=\frac{1}{2}\left(z_{1} \partial_{z_{1}}-z_{2} \partial_{z_{2}}+\partial_{\bar{z}_{1}} \bar{z}_{1}-\partial_{\bar{z}_{2}} \bar{z}_{2}\right) \text {, } \\
& \mathbf{J}_{\zeta \bar{\zeta}}=\frac{1}{2}\left(z_{1} \partial_{z_{1}}-z_{2} \partial_{z_{2}}-\partial_{\bar{z}_{1}} \bar{z}_{1}+\partial_{\bar{z}_{2}} \bar{z}_{2}\right) \\
& C_{1}=z_{1} \partial_{z_{1}}+z_{2} \partial_{z_{2}}-\partial_{\bar{z}_{1}} \bar{z}_{1}-\partial_{\bar{z}_{2}} \bar{z}_{2} .
\end{aligned}
$$

Now we link the variables $p_{v}$ and $\zeta_{i}$ with $z_{1}$ and $z_{2}$ as follows:

$$
\begin{gathered}
\zeta=\mathrm{i} \frac{z_{1}}{z_{2}}, \quad p_{v}=z_{2} \bar{z}_{2}, \quad \phi=\frac{\mathrm{i}}{2} \ln \frac{\bar{z}_{2}}{z_{2}}, \\
p_{v}>0, \quad \phi \in[0,2 \pi],
\end{gathered}
$$

where we again use the complex variable $\zeta$ given by (2.2) and introduce an additional variable $\phi$. Note that in contrast to Minkowski space-time (see Ref.[20]), where $z_{i}$ are linked with lightlike momentum $p_{\mu}$ in our case $z_{i}$ are linked with momentum $p_{v}$ as well as space coordinates $\zeta_{i}$. It is clear that the function $f\left(z_{1}, z_{2}\right)$ can be rewritten as a certain function of new variables $\Phi\left(p_{v}, \zeta_{i}, \phi\right)$ :

$$
f\left(z_{1}, z_{2}\right)=\Phi\left(\zeta_{i}, p_{v}, \phi\right) .
$$

The corresponding measure from (5.3) in terms of new variables is given by

$$
d^{2} z_{1} d^{2} z_{2}=p_{v} d^{2} \zeta d p_{v} d \phi
$$

Making use of chain rules

$$
\partial_{z_{1}}=\frac{\mathrm{i}}{z_{2}} \partial_{\zeta}, \quad \partial_{z_{2}}=\frac{1}{z_{2}}\left(-\zeta \partial_{\zeta}+\mathrm{i} p_{v} v-\frac{\mathrm{i}}{2} \partial_{\phi}\right)
$$

and their analog for $\partial / \partial \bar{z}$ one gets the following representations for generators acting on the functions $\Phi\left(p_{v}, \zeta_{i}\right)$ :

$$
\begin{aligned}
\mathbf{J}_{i j} & =-\zeta_{i} \partial_{j}+\zeta_{j} \partial_{i}+\frac{1}{2} \epsilon_{i j} \partial_{\phi}, \\
\mathbf{J}_{v i} & =-\mathrm{i} \zeta_{i} p_{v}, \\
\mathbf{J}_{u v} & =-\mathrm{i} v p_{v},
\end{aligned}
$$




$$
\begin{aligned}
& \mathbf{J}_{u i}=v \partial_{i}+\frac{\mathrm{i}}{2 p_{v}} \zeta_{i} \partial^{2}-\frac{\mathrm{i}}{2 p_{v}} \epsilon_{i j} \partial_{j} \partial_{\phi}, \\
& ( \pm) \mathbf{K}_{u}=v \mathbf{D}+\frac{\mathrm{i}}{4 p_{v}} \zeta^{2} \partial^{2}-\frac{\mathrm{i}}{2 p_{v}} \zeta_{i} \epsilon_{i j} \partial_{j} \partial_{\phi}+\frac{\mathrm{i}}{4 p_{v}} \partial_{\phi}^{2} \\
& ( \pm) \mathbf{K}_{v}=-\frac{\mathrm{i}}{2} \zeta^{2} p_{v} \\
& ( \pm) \mathbf{K}_{i}=-\zeta_{i} \mathbf{D}+\frac{1}{2} \zeta^{2} \partial_{i}+\frac{1}{2} \epsilon_{i j} \zeta_{j} \partial_{\phi} \\
& \mathbf{D}=-\mathrm{i} v p_{v}+\zeta_{j} \partial_{j}+1 \\
& C_{1}=-\mathrm{i} \partial_{\phi}-2
\end{aligned}
$$

where in the last expressions we go back to $\zeta_{i}$ [see (2.2)]. Note that for $\mathbf{P}_{\mu}$ the results are the same as in (4.45). Now expand $\Phi\left(p_{v}, \zeta_{i}, \phi\right)$ in Fourier series with respect to $\phi$ over the interval $[0,2 \pi]$ :

$$
\Phi\left(p_{v}, \zeta_{i}, \phi\right)=\frac{1}{\sqrt{2 \pi}} \sum_{\lambda=0, \pm \frac{1}{2}, \pm 1, \ldots} \Phi_{\lambda}\left(p_{v}, \zeta_{i}\right) e^{2 \mathrm{i} \lambda \phi} .
$$

Note that each term under sum in (5.6) transforms into an irreducible representation of $u(2,2)$ for which $C_{1}$ is diagonal and takes the values

$$
C_{1}=2(\lambda-1), \quad \lambda=0, \pm \frac{1}{2}, \pm 1, \pm \frac{3}{2}, \ldots
$$

where

$$
\partial_{\phi}=2 \mathrm{i} \lambda
$$

By substituting (5.8) into (5.5) and (4.47) into (4.45) we take one and the same expression. Thus we have proved completely that by decomposing the ladder representation into irreducible representations of the $u(2,2)$ algebra, we get a chain of plane wave geometry massless states with helicities displayed in (5.7) where each state is included just once. The corresponding norm is also decomposed into direct sum as

$$
(\Phi, G)=\sum_{\lambda} \int p_{v} d p_{v} d^{2} \zeta \overline{\Phi_{\lambda}\left(p_{v}, \zeta\right)} G_{\lambda}\left(p_{v}, \zeta\right)
$$

At the end of this section we would like to demonstrate how Minkowski and plane wave geometry massless fields are related to each other. A reason for such an interrelation comes from the fact that chains of Minkowski as well as plane wave geometry massless fields realize the same commutation relations (5.1). To demonstrate such interrelation explicitly let us recall one of the results of Ref.([20]). In Ref.([20]) it was shown that for the case of Minkowski massless fields it is necessary to choose the following representation for operator-valued four-component spinor $\varphi_{\text {Min }}$ 


$$
\varphi_{\text {Min }}=\left(\begin{array}{c}
\lambda_{\text {Min }} \\
\pm \tau_{\text {Min }}
\end{array}\right), \quad \lambda_{\text {Min }}=\left(\begin{array}{c}
\bar{z}_{1} \\
\bar{z}_{2}
\end{array}\right), \quad \tau_{M i n}=\left(\begin{array}{c}
\partial_{z_{1}} \\
\partial_{z_{2}}
\end{array}\right) .
$$

Please compare with our choice for the case of plane wave massless fields (5.4). Now what needs to be demonstrated is that $\varphi$ and $\varphi_{\text {Min }}$ are related by unitary transformation

$$
\varphi=U \varphi_{\text {Min }},
$$

where the matrix $U \in U(2,2)$-group, i.e., it should satisfy

$$
U^{*} \beta U=\beta .
$$

Our statement is that such a intertwine matrix exists and is given by

$$
U=\left(\begin{array}{cccc}
0 & 0 & \pm 1 & 0 \\
0 & 1 & 0 & 0 \\
\pm 1 & 0 & 0 & 0 \\
0 & 0 & 0 & 1
\end{array}\right)
$$

\section{Conclusion}

We have constructed free equations of motion for plane wave geometry massless fields of arbitrary spin. We believe that this result can get interesting development and applications. Let us briefly outline the following obvious applications. First of all, the equations in question could be easily generalized to five-dimensional plane wave geometry. Therefore, making use of the procedure of dimensional reduction, one could get a description of four-dimensional massive arbitrary spin fields. From this point of view this result can get applications in systematical analysis of string massive states spectrum. Another interesting potential application our result can get is the problem of introducing an interaction into the theory of massless higher spin fields. Note that for the case of anti-de Sitter geometry this problem, at least at classical level, has been solved recently in Ref.([22]). One can speculate that for the case of plane wave geometry higher massless spin fields this problem also has a positive solution. Note that in Ref.([22]) it was demonstrated that it is the spectrum of anti-de Sitter massless states related to the $u(2,2)$ algebra ladder representation that leads to self-consistent interaction of anti-de Sitter massless higher spin fields. Therefore it is strongly believed that our results related to ladder representations can also get interesting applications in the problem of interaction of plane wave geometry massless higher spin fields. Because detailed investigation of these questions is too involved, we hope to study them in future publications.

\section{Acknowledgements}

The author wishes to thank M.A. Vasiliev for useful suggestions. This work was supported in part by INTAS, Grant No.94-2317, by the Russian Foundation for Basic Research, Grant No.96-01-01144, and by the NATO Linkage, Grant No.931717. 


\section{Appendix A. Spin part of conformal algebra}

For the case of the vector field $(s=1)$ the Lie derivative

$$
\mathcal{L}_{\xi} \Phi^{\mu_{1}, \ldots \mu_{s}}=\xi^{\nu} \partial_{\nu} \Phi^{\mu_{1} \ldots \mu_{s}}-\sum_{k=1}^{s} \Phi^{\mu_{1} \ldots \nu \ldots \mu_{s}} \partial_{\nu} \xi^{\mu_{k}}
$$

can be rewritten in terms of $\Phi^{A}[$ see $(2.10)]$ as follows:

$$
\mathcal{L}_{\xi} \Phi^{A}=\xi^{\mu} \partial_{\mu} \Phi^{A}-e_{B}^{\mu} \Phi^{B} \partial_{\mu} \xi^{A}+\delta_{V}^{A} F(u)\left(\xi^{U} \zeta^{i} \Phi^{i}-\Phi^{U} \zeta^{i} \xi^{i}\right),
$$

where we introduce $\xi^{A}=\mathbf{e}_{\mu}^{A} \xi^{\mu}$. Now it is clear how the last expression can be generalized to the case of arbitrary rank tensor field. Making use of generating function [see 2.12] the Lie derivatives can be written as

$$
\mathcal{L}_{\xi}|\Phi\rangle=G|\Phi\rangle
$$

where

$$
G=\xi^{\mu} \partial_{\mu}+e_{A}^{\mu} \partial_{\mu} \xi^{B} a_{B} \bar{a}^{A}+a_{V} \zeta^{i} F(u)\left(-\xi^{U} \bar{a}_{i}+\xi^{i} \bar{a}_{V}\right) .
$$

The first term on the rhs corresponds to the orbital part and shall be denoted by $l(G)$, while the rest is called spin part and shall be denoted by $s(G)$. Now we are going to rewrite $s(G)$ in a more invariant manner, namely in terms of $M_{A B}$ [see (2.14)] which, in a space of totally symmetric tensor fields (2.12), has the following representation:

$$
M_{A B}=-a_{A} \bar{a}_{B}+a_{B} \bar{a}_{A} .
$$

Making use of (3.1) one can make sure that the following relations hold true:

$$
\partial_{v} \xi^{i}=\partial_{i} \xi^{U}, \quad e_{U}^{\mu} \partial_{\mu} \xi^{V}=-F(u) \zeta^{i} \xi^{i}, \quad \partial_{v} \xi^{U}=0
$$

and

$$
-e_{U}^{\mu} \partial_{\mu} \xi^{i}+\partial_{i} \xi^{V}=\zeta^{i} \xi^{U}, \quad e_{U}^{\mu} \partial_{\mu} \xi^{U}+\partial_{v} \xi^{V}=\frac{1}{2}(\nabla \xi) .
$$

With the help of these relations and (A.1), the expression for $s(G)$ can be rewritten as

$$
\begin{aligned}
s(G) & =-\partial_{v} \xi^{V} M_{V U}+\left(\partial_{i} \xi^{V}-F(u) \zeta^{i} \xi^{U}\right) M_{V i}+\partial_{v} \xi^{i} M_{U i} \\
& +\frac{1}{4}(\nabla \xi)\left(\Delta+2 M_{V U}\right)-\frac{1}{2} \partial_{i} \xi_{j} M_{i j}
\end{aligned}
$$

where

$$
\Delta=2 a_{V} \bar{a}_{U}-a_{i} \bar{a}_{i}
$$

Note that while deriving $s(G)$ we taken into account only (3.1). Taking into account equations for isometry Killing vector $\nabla_{\mu} \xi_{\nu}+\nabla_{\nu} \xi_{\mu}=0$ we arrive at (2.13). It is clear that the last representation for $s(G)$ is valid not only for totally symmetric tensor fields but for those of arbitrary symmetry as well. 


\section{Appendix B. Superalgebra osp $(2,1)$}

In this Appendix we prove the following interesting statement: if $y_{\alpha}$ and $Y_{a}$ are independent solutions of the equations (3.5) and (3.4) respectively then the algebra (3.7), where the brackets are defined by (3.6), is isomorphic to superalgebra $\operatorname{osp}(2,1)$. To prove the statement we choose the following solutions of Eq.(3.4):

$$
Y_{1}=y_{1}^{2}, \quad Y_{2}=y_{2}^{2}, \quad Y_{3}=y_{1} y_{2}
$$

For such a choice of $Y_{a}$ the determinant of Eq.(3.4) is equal to $-2 W^{3}$, where $W$ is a determinant of (3.5): $W=y_{1} y_{2}^{\prime}-y_{2} y_{1}^{\prime}$. Note that $W=$ const. Because $y_{\alpha}$ form independent solutions of (3.5), i.e., $W \neq 0$, the $Y_{a}$ in (A.1) also form independent solutions of Eq.(3.4). Now the statement can be proved by direct calculation. Actually, making use of (B.1) and (3.6), it is easy to derive the following commutation relations:

$$
\begin{gathered}
\left\{J_{0}, J_{ \pm}\right\}= \pm J_{ \pm}, \quad\left\{J_{+}, J_{-}\right\}=-2 J_{0}, \\
\left\{J_{ \pm}, R_{\mp}\right\}=\mp R_{ \pm}, \quad\left\{J_{0}, R_{ \pm}\right\}= \pm \frac{1}{2} R_{ \pm}, \\
\left\{R_{ \pm}, R_{ \pm}\right\}=J_{ \pm}, \quad\left\{R_{+}, R_{-}\right\}=J_{0},
\end{gathered}
$$

where the notation $J_{0}=Y_{3} / W, J_{+}=Y_{2} / W, J_{-}=Y_{1} / W, R_{+}=y_{2}$ and $R_{-}=y_{1}$ has been used. The relations (B.2)-(B.4) are nothing but the commutation relations of $o s p(2,1)$ superalgebra. The statement is proved. 


\section{References}

[1] H.W. Brinkman, Proc. Natl. Acad. Sci. (U.S.) 9, 1 (1923); N. Rosen, Phys. Z. Sowjetunion 12, 366 (1937).

[2] H. Bondi, F.A. Pirani and I. Robinson, Proc. Roy. Soc. (London) A 251, 519, (1959).

[3] R. Penrose, Rev. Mod. Phys. 37, 215 (1965).

[4] S. Deser, J. Phys. A 8, 1972 (1975).

[5] G.W. Gibbons, Comm. Math. Phys. 45, 191 (1975).

[6] C.R. Nappi and E. Witten, Phys. Rev. Lett. 71, 3751 (1993).

[7] G. Horowitz and A. Steif, Phys. Rev. Lett. 64, 260 (1990); Phys. Rev. D 42, 1950 (1990).

[8] G.T. Horowitz and A.A. Tseytlin, Phys. Rev. D 50, 5204 (1994).

[9] A.A. Tseytlin, Phys. Rev. D 473421 (1993); K. Sfetsos and A.A. Tseytlin, Nucl. Phys. B 427, 245 (1994).

[10] A.A. Kehagias and P.A.A. Meessen, Phys. Lett. B 331, 77 (1994).

[11] I. Antoniadis and N.A. Obers, Nucl. Phys. B 423, 639 (1994).

[12] P. Forgacs, P.A. Horvathy, Z. Horvath and L. Palla, The Nappi-Witten string in the light cone gauge, hep-th/9503222, in Ahrenshoop Symposium, 324-338, (1994).

[13] V. Bhansali, J. Math. Phys. 32, 302 (1991).

[14] W. Siegel, Int. J. Mod. Phys. 4, 2015 (1989).

[15] R.R. Metsaev, Mod. Phys. Lett. A 10, 1719 (1995).

[16] Handbook of Mathematicial Functions, Eds M. Abramowitz and I.A. Stegun, in: Applied Mathematical Series 55. National Bureau of Standards. Washington: Government Printing Office, 1964.

[17] C. Fronsdal, Phys. Rev. D 12, 3819 (1975); Phys.Rev. D 20, 848 (1979).

[18] N.T. Evans, J. Math. Phys. 8, 170 (1967).

[19] P. Breitenlohner and D.Z. Freedman, Ann. Phys. 144, 249 (1982).

[20] G. Mack and I. Todorov, J. Math. Phys. 10, 2078 (1969).

[21] C. Fronsdal, Preprint UCLA /85/TEP/10, Massless particles, orthosymplectic symmetry and another type of Kaluza-Klein theory.

[22] M.A. Vasiliev, Phys. Lett. B 243, 378 (1990); Phys. Lett. B 257, 111 (1991); Class. Quantum Grav, 8, 1387 (1991).

[23] D.Tz. Stoyanov and I.T. Todorov, J. Math. Phys. 9, 2146 (1968). 